\title{
The Theoretical Study of Photoemission from Various Region of Metal and Semiconductor
}

\author{
Gopi Chandra Kaphle \\ Central Department of Physics, Tribhuvan University, Kirtipur, Kathmandu, Nepal \\ gck223@gmail.com
}

\begin{abstract}
This study involves the theoretical analysis of photoemission from the surface of metal(Al) and semiconductor (Si) in various regions. For the analysis of Photoemission, we calculated the vector potential with the help of dielectric model provided by Bagchi and Kar in modified form. From the calculation it is observed that electrons are mostly exited effectively at the surface regions than that of vacuum and bulk indicating that Photoemission takes place from the surface region, which favors the well accepted experimental and theoretical results. This study can be used to calculate photocurrent by the help of band structure of metals and semiconductors.
\end{abstract}

Keywords: photo-field emission, dielectric model, vector potential

\section{INTRODUCTION}

Photoemission spectroscopy(PES)is the most popular, simple and important ethod for studying the electronic structure of atom of the variety of the substances of solids. Nowadays the application of this technique can be considered as the study of solids at the bulk and near the surface. The detailed information related to the band structure of crystal, the lifetimes of electrons and holes in solids, the geometrical orientation and ordering of the valence energy levels of atoms and molecules absorbed on surface etc can be extracted from the photoemission. Photoelectron emission involves the emission of electrons from matter, in response to photons being absorbed by it. During this process, part of the radiation incident on the solids is absorbed and part of the radiation is reflected. It is also follows the consevation of energy of the electron such thatEf$\mathrm{Ei}={ }^{-} \mathrm{h}$ ! where ${ }^{-} \mathrm{h}$ ! is the energy of the incident photon.This was firstly predicted by Einstein and nowadays covers wide fields of research. Photoelectron spectroscopy, X-ray photoelectron spectroscopy(XPS), Ultraviolet photoelectron spectroscopy (UPS) and extreme ultraviolet photoelectron spectroscopy(EUPS) lies in between XPS and UPS etc are mostly used to study the valence energy levels, chemical bonding and valence band structure calculations. UPS is used for the better energy resolution and faster ejection of electrons for better spectrum signals.
In this case we used angle resolved ultraviolet photoemission spectroscopy (ARUPS) as a theoretical base because it measures and analyzes the energy of electrons emitted at the particular angle and collects the energy in hemispherical analyzer. Feibelman [1] introduced a realistic model called random phase approximation and used his theory for the jellium model and found that datas are good agreement with Levinson et.al [2] but it suffers from its inapplicability to other metal sand fields.Mele and Joannopoules present a microscopic model for the formation of schottky barriers at metals-semiconductors contacts. Kempa and Forstman [3] performed detailed calculation of eltromagnetic fields using hydrodynamical model and used it in Aluminium, which shows that the frequency dependence of surface photoemission yield is due to behavior of electric field. Ishii and Aisaka [4] presented theoretical analysis of ARUPS by using the dynamical multiple scattering formalism for photoelectron. Maniv and Mathieu [5] made considerable progress in the calculation of electromagnetic field in metal vacuum interfacial region.

Bagchi and Kar [6, 7] employed local dielectric function inside the crystal and vacuum. R. K. Thapa and Coworkers $[8,9]$ have done effective theoretical study on photoemission and photo-field emission and applicable to electronic properties of metals and semiconductors. Actually these all the results 
motivated us to work in the fields of photoemission and photo-field emission. However due to limitation of computational and other technical problem we here only deal with vector potential calculation and its effect in Bulk, surface and Vac uum of metal and semiconductor.

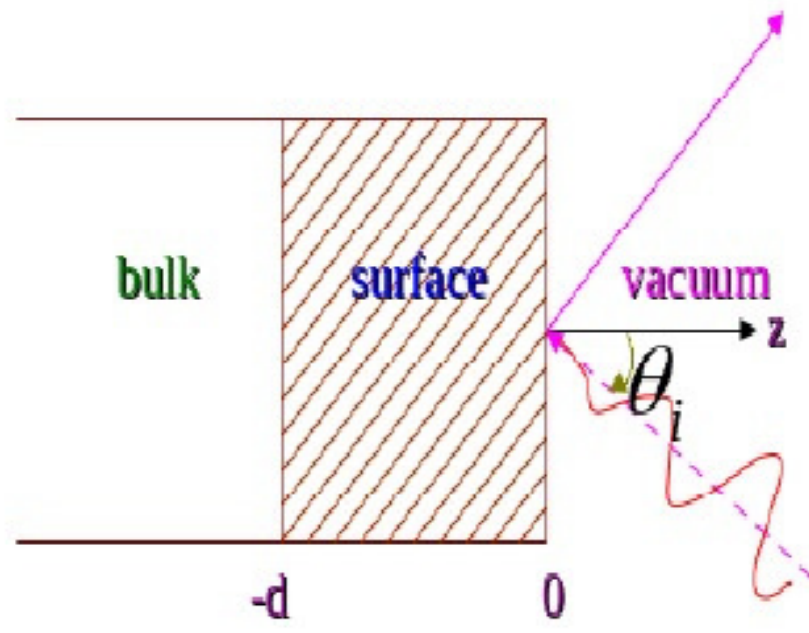

Fig.1 Model of dielectric for the calculation of vector potential

For the calculation of electromagnetic field,the dielectric model gi ven in fig(1)is taken as a reference. At which we assumed that metal or semiconductor is placed in the space to the left of $\mathrm{z}=0$ plane such that dielectric value varies linearly over the region $-\mathrm{d}<\mathrm{z}<0$, where it is a local function interpolating between the bulk value inside the metal and vacuum value (unity) outside.The frequency dependent dielectric model therefore be written as,

$$
\varepsilon(\omega, z)=\left\{\begin{array}{ll}
\varepsilon_{1}(\omega)+i \varepsilon_{2}(\omega) & \text { if } z \leq-d \\
1+[1-\varepsilon(\omega)] \frac{z}{d} & \text { if }-d \leq z \leq 0 \\
1 & \text { if } z \geq 0
\end{array}\right\}
$$

where $\mathrm{d}$ is the width of the surface and $\varepsilon(\omega)$ is the frequency dependent complex dielectric function. The refractive index of bulk metal is also a complex such that

$$
\mathrm{n}=\varepsilon 1+\mathrm{i} \varepsilon 2=\mathrm{n}+\mathrm{i} \kappa \text {. }
$$

where $\varepsilon 1(\omega)$ and $\varepsilon 2(\omega)$ are real and imaginary parts of the dielectric function. For the photoemission, the light wave having angular frequency h $\omega$ is taken as the incident radiation which is incident with $(\theta \mathrm{i})$ as a angle of incidence on the metal surface defined by $x-y$ plane. We actually consider that photoe-. We actually consider that photoemission takes place along z-axis, which is normal to the surface as in fig.(1) given by $z=0$ plane. We may therefore write $\mathrm{H}$ as a,

$H=\left(\frac{e}{m c}\right)\left[A_{\omega}(z) \frac{d}{d z}+\frac{1}{2} \frac{d}{d z} A_{\omega}(z)\right]$

where A $\omega(\mathrm{z})$ is vector potential. A model of Bagchi and Kar is used to calculate it.For it, A gauss was chosen in such a way that it sets the scalar potentiato zero. The setting of zero scalar potential helps to express electromagnetifield in terms of vector potential as,

$\vec{E}(k, \omega, z)=\frac{i \omega}{c} \vec{A}(k, \omega, z)$

where $\mathrm{A}(\mathrm{k}, \omega, \mathrm{z})$ is the vector potential and $\mathrm{k}=\omega /$ $\operatorname{csin}(\theta \mathrm{i})$ is the parallel component of wave vector. Now for the calculation of vector potential we will take the help of Maxwell's fundamental equations at which magnetic fields in terms of vector potential can be expressed as,

$\vec{B}=\Delta \times \vec{A}$

such that $\mathrm{A}(\mathrm{r}, \mathrm{t})=\mathrm{A}(\mathrm{z}) \operatorname{expi}\left(\mathrm{K} . \rho^{-}-\omega \mathrm{t}\right)$ where $\rho=\mathrm{x}^{\wedge}$ $+\mathrm{y} y$ and $\mathrm{K}=\mathrm{k}^{\wedge}=\mathrm{c} \sin (\theta \mathrm{i})^{\wedge}$. Using the boundary condition and solving Maxwell's equation at the surface level vector potential can be obtained as,

$\Delta(\Delta . A)-\Delta^{2} A=\frac{-\varepsilon}{c^{2}} \frac{\partial^{2} A(z)}{\partial t^{2}}$

and with straightforward calculation above equation will takes the form,

$\left(-k^{2}+\frac{\partial^{2}}{\partial t^{2}}\right) A(z)+\frac{\varepsilon \omega^{2}}{c^{2}} A(z)-\left(i k \hat{x}+\frac{\partial}{\partial z} \hat{z}\right)\left[\left(i k \hat{x}+\frac{\partial}{\partial z} \hat{z}\right) \cdot A(z)\right]=0 \quad(6)$

This is the main equation to evaluate the vector potential.which is obtained either from s-polarization or p-polarization cases.

In former case vector potential A is parallel to $\mathrm{y}$-axis so the electric field i.e. incident wave is parallel to y-axis. So we can not observe any effect of electromagnetic radiation emission. It is mainly due to the fact that detector cannot detect such types of emission properly. Hence we use only p-polarization cases for the calculation of vector potential and electromagnetic effect on the surface of metals and semiconductors. 
In p-polarization cases electromagnetic vector potential A is in the $\mathrm{x}-\mathrm{Z}$ plane and magnetic field is parallel to $y$-axis. Hence $x$ and $y$ components of vector potential can be deduced from the equation(5) as,

$$
\frac{d^{2} A_{x}}{d z^{2}}+\frac{\varepsilon \omega^{2}}{c^{2}} A_{x}=i k \frac{d A_{z}}{d z}
$$

where $A^{x}(z) \hat{x}=A_{x}$ and $A^{x}(z) \hat{z}=A_{z}$

and

$$
\left(\frac{\varepsilon \omega^{2}}{c^{2}}-K^{2}\right) A_{z}=i k \frac{d A_{x}}{d z}
$$

where $A^{z}(z) \hat{x}=A_{x}$ and $A^{z}(z) \hat{z}=A_{z}$

using little algebra and equating results into Landau and Lifshitz equation [10] we obtained new equation in terms of B as,

$\frac{\partial}{\partial z}\left(\frac{1}{\omega} \frac{\partial B}{\partial z}\right)+\left(\frac{\omega^{2}}{c^{2}}-\frac{K^{2}}{\varepsilon}\right) B=0$

Wnich is solved under the boundary condition that both $\mathrm{B}$ and $\partial \mathrm{B} / \mathrm{dz}$ are continious.

Now using $B(z)=u(z) \sqrt{\varepsilon(z)}, \frac{\omega^{2}}{c^{2}}=k^{2}$ and $K=k \sin \left(\theta_{i}\right)$ then the common electromagnetic equation can be obtained as,

$$
\frac{d^{2} u}{d z^{2}}+k^{2}\left(\varepsilon-\sin ^{2} \theta_{i}\right) u+\left[\frac{1}{2 \varepsilon} \frac{d^{2} \varepsilon}{d z^{2}}-\frac{3}{4} \frac{1}{\varepsilon^{2}}\left(\frac{d \varepsilon}{d z}\right)^{2}\right] u=0
$$

Now substituting the values of dielectric for each region from equation(1) and solving equation we obtained the magnetic vector potential as,

$$
\vec{A}_{\omega}(z)=\left\{\begin{array}{ll}
\frac{-\varepsilon \sin 2 \theta_{i}}{\left[\varepsilon-\sin ^{2} \theta_{i}\right]^{\frac{1}{2}}+\varepsilon \cos \theta_{i}} & \text { for } z \leq 0 \\
\frac{-\sin 2 \theta_{i}}{\left[\varepsilon-\sin ^{2} \theta_{i}\right]^{\frac{1}{2}}+\varepsilon \cos \theta_{i}} \frac{\varepsilon d}{d+(1-\varepsilon) z} & \text { for }-d \leq z \leq 0 \\
\frac{-\sin 2 \theta_{i}}{\left[\varepsilon-\sin ^{2} \theta_{i}\right]^{2}+\varepsilon \cos \theta_{i}} & \text { for } z \leq-d
\end{array}\right\}
$$

For the explanation of the photoelectric emission, equation(10) has to be plotted $|\mathrm{A} \omega(\mathrm{z})| 2 \mid$ against the photon energy $(-\omega)$ at various region or $\mathrm{dA} \omega / \mathrm{dtverses}$ distance at various radiation energy. For the use of computational purpose data from the references $[11,12]$ are taken as standard. The surface "width for metals and semiconductors are taken as 10A.

\section{CALCULATION AND EVALUATION OF FIELD VECTOR}

The s-polarization is not taken into account for the photoemission, as light doesn't play an effective role for it, which is also one of the evident from the calculation of Weng et al.So, here we only explain the result coming from the plot of electromagnetic field i.e. vector potential $|\mathrm{A} \omega(\mathrm{z})| 2 \mid$ against photon energy h $\omega$ or distance ( $\mathrm{z}$ ) at vacuum, surface and bulk region, which help us to find out the electromagnetic behavior of metals and semiconductors.
In case of Aluminum peak is obtained at $11 \mathrm{ev}$ at the surface and minimum peak occur at $15 \mathrm{ev}$ where as such behavior is not observed in the other bulk and vacuum cases. The minimum value in the field was expected at the plasmon energy hwp of Aluminum i.e $15.8 \mathrm{ev}$, but in our case it is slightly deviated. Peak value in the field at $11 \mathrm{ev}$ as in fig(2) is due to the excitations of electrons of surface plasmon which is related with bulk plasmon by $h \omega s=\sqrt{\omega} / 2$.

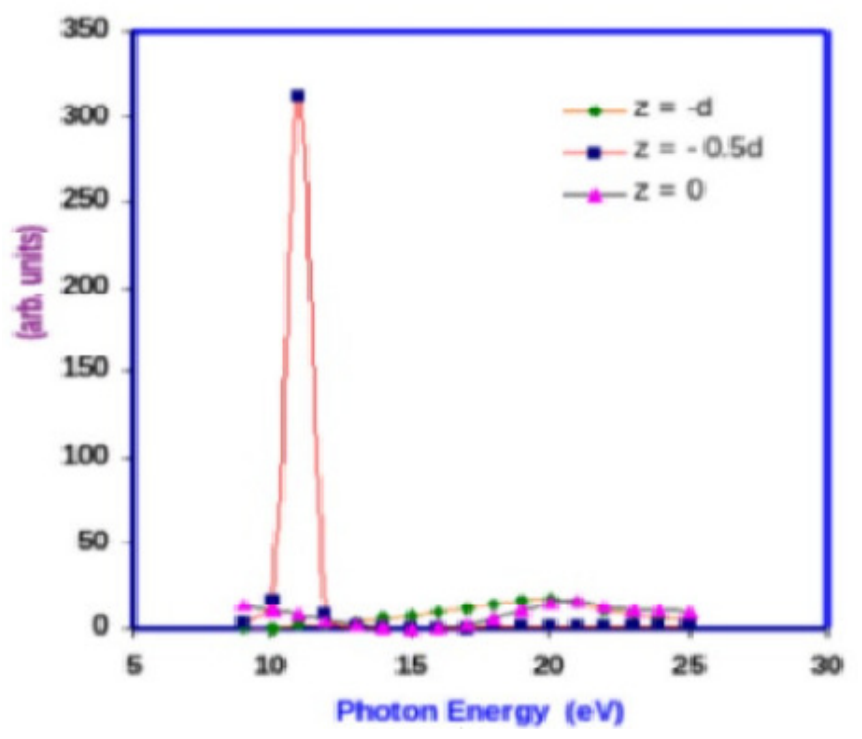

Fig 2. Variation of $|\mathrm{A} \omega(\mathrm{z})| 2$ against photon energy for $\mathrm{Al}$

We have also plotted graph of the field against the distance $(\mathrm{z})$ for different

values of photon energy as in fig(3),

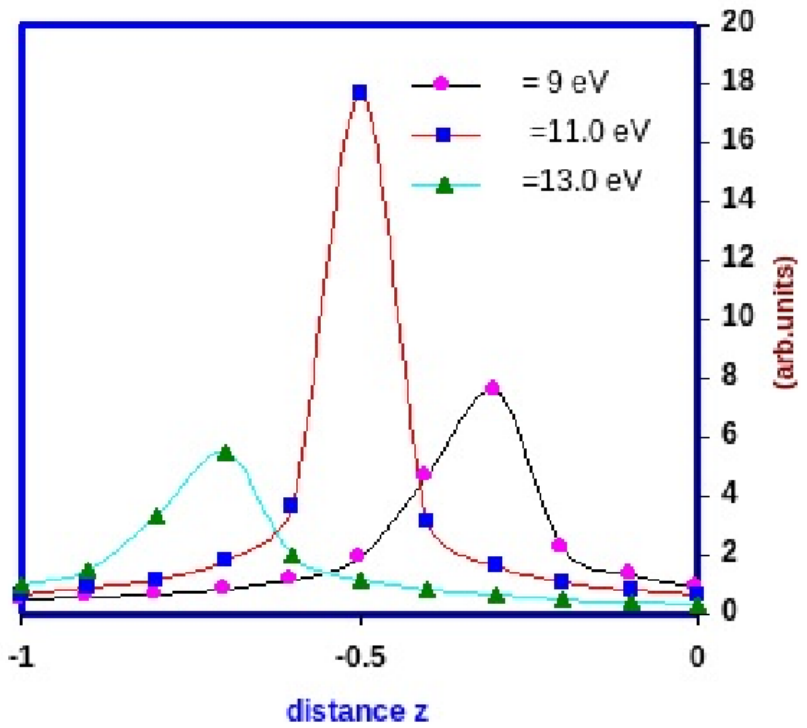

Figure 3: Plot of ( dA $\omega)$ /dz against distance at various photon energy in $\mathrm{Al}$ 
It has been observed that highest peak is obtained at $11 \mathrm{ev}$ when $\mathrm{d}=-0.5$ i.e at the surface region. This indicates that maximum emission takes place from the surface of $\mathrm{Al}$.

Similarly from the plot of electromagnetic field $|A \omega(\mathrm{z})| 2$ verses photon energy ${ }^{-} \mathrm{h} \omega$, it is observed that strong peak is occurred at $7.0 \mathrm{ev}$ contrast to the other region where as minima is observed at 12.8 ev.for the surface region of Si.

Other peak is at $16.2 \mathrm{ev}$. This is the position at which dielectric function shows a resonance behavior. Same behavior is in the case of bulk region but maxima occurred at $7.8 \mathrm{ev}$. However in the vacuum region the maximum value is at $12.8 \mathrm{ev}$ and minima occurs at the plasmon energy i.e $16.2 \mathrm{ev}$. This is the contrast to the behavior of the field in the bulk surface boundary plane.

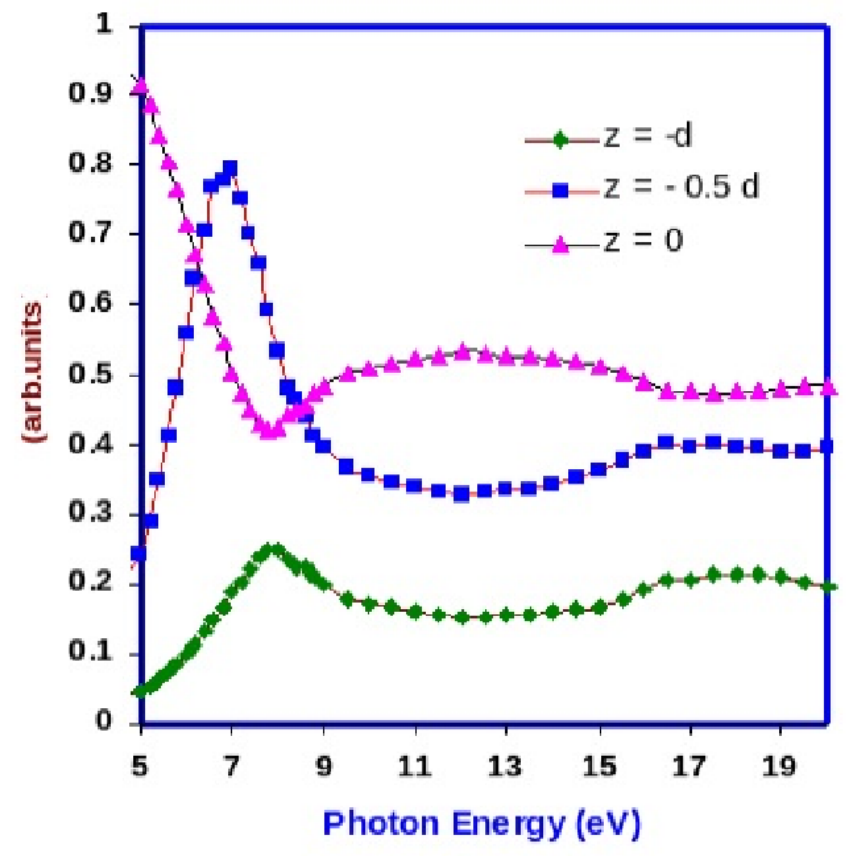

Figure 4: Plot of $|\mathrm{A \omega}(\mathrm{z})| 2$ against photon energy for $\mathrm{Si}$

\section{CONCLUSIONS}

This paper gives the theoretical study of calculation of electromagnetic field of Aluminum (Al) and semiconductor Silicon (Si) using the model provided by Bagchi and Kar in modified form. For the calculation of electromagnetic field, we analyze the variation of $|A(\omega)(z)| 2$ with respect to h $\omega$ i.e. photo energy, which provides the information of emission of electrons from the surface of metals and semiconductors.

In the case of $\mathrm{Al}$ the peak value of electromagnetic field is found minimum at the plasmon energy but it is deviated slightly due to the fact that peak value of excitation of related to bulk. This indicates that both surface and bulk provides the photoemission effect. But from figure, we conclude that electromagnetic field is maximum at the surface region i.e. peak value obtained at $h \omega=11 \mathrm{eV}$ at $\mathrm{z}=-0.5 \mathrm{~d}$.

In case of $\mathrm{Si}$, we got different peak but there is resonance at $7.8 \mathrm{eV}$, i.e. due to narrow surface width, the emission of photocurrent is negligible for all values of photon energy. This means dielectric function for silicon shows a resonance in contrast to behavior of field in the bulk-surface boundary plane. This indicates that the variation of field in the surface region of semiconductor is very important in case of photoemission. Similar kinds of result is shown previously by one of us [13].

Finally with the above study and analysis,following conclusions can be drawn as,

1. The metal (Al) and semiconductor (Si) show the electromagnetic properties at the surface region i. e. maximum number of photoelectrons emitted from the surface region.

2. The photoelectric effect can be studied after the calculation of electromagnetic fields or vector potentialAw $(\mathrm{z})$ inside the metals and semiconductor.

3. This theory can be extended to calculate the photocurrent and photo field emission which is useful for the band structure calculations of metals and semiconductors.

\section{ACKNOWLEDGEMENTS}

I am grateful to Mr. Bandan Chakraborty of S.N.Bose National Center for basic sciences, kolkata for technical discussion and Prof. R. K. Thapa of Mizoram University for providing necessary articles and program for the work. 


\section{REFERENCES}

[1] A. Bachi and N. Kar, Phy. Rev. B18, 5240 (1978)

[2] A. Bachi ,Phy. Rev. B5, 3060 (1977)

[3] A. Ishi, T .Aissaka, Surface Science, 242, 250 (1990)

[4] G. C. Kaphle, Study of the behavior of electromagnetic fields of metals and semiconductors", Mini Research submitted to U. G. C. Nepal, Sanothimi Bhaktapur.

[5] H. T. Levinson et'. al, Phy. Rev. Lett., 43, 952 (1979)

[6] J. H. Weaver, Handbook of Chemistry and physics, CRC press, Boca Raton F L, 1987, PE 377
[7] Kittel, Charles: "Introduction to solid states physics", 8th edition, willey 2005

[8] K. Kempa and Forstman, Surface science, 129, $516(1983)$

[9] L. D. Landau and E. M. Lifshitz: Electrodynamics of Contineous media. (Pergamon, Newyork, 1980)

[10] P. J. Feibelman, Phy. Rev. B12, 1319 (1975)

[11] R. K. Thapa, P. Das and N. Kar, Mod. Phy. Lett. B8, 36 (1994)

[12] R. K. Thapa and N.Kar, Phy. Rev. B, 51. 24 (1995)

[13] T. Maniv and H. Matieu, Phy. Rev. B22, 4731 (1980) 